\title{
Biophysical properties of Saccharomyces cerevisiae and their relationship with HOG pathway activation
}

\author{
Jörg Schaber • Miquel Àngel Adrover · Emma Eriksson · Serge Pelet • \\ Elzbieta Petelenz-Kurdziel • Dagmara Klein - Francesc Posas • \\ Mattias Goksör • Mathias Peter · Stefan Hohmann • Edda Klipp
}

Received: 11 January 2010/Revised: 18 March 2010/ Accepted: 12 May 2010/Published online: 19 June 2010

(C) The Author(s) 2010. This article is published with open access at Springerlink.com

\begin{abstract}
Parameterized models of biophysical and mechanical cell properties are important for predictive mathematical modeling of cellular processes. The concepts of turgor, cell wall elasticity, osmotically active volume, and intracellular osmolarity have been investigated for decades, but a consistent rigorous parameterization of these concepts is lacking. Here, we subjected several data sets of minimum volume measurements in yeast obtained after hyper-osmotic shock to a thermodynamic modeling framework. We estimated parameters for several relevant biophysical cell properties and tested alternative hypotheses
\end{abstract}

M. À. Adrover, E. Eriksson, S. Pelet, E. Petelenz-Kurdziel have contributed equally to this work.

Electronic supplementary material The online version of this article (doi:10.1007/s00249-010-0612-0) contains supplementary material, which is available to authorized users.

J. Schaber · E. Klipp $(\bowtie)$

Theoretical Biophysics, Humboldt University, Invaliden Str. 42, 10115 Berlin, Germany

e-mail: edda.klipp@rz.hu-berlin.de

M. À. Adrover · F. Posas

Cell Signaling Unit, Departament de Ciències Experimentals i de la Salut, Universitat Pompeu Fabra (UPF),

08003 Barcelona, Spain

E. Eriksson · M. Goksör

Department of Physics, University of Gothenburg,

41296 Gothenburg, Sweden

S. Pelet $\cdot$ M. Peter

Institute of Biochemistry, ETH, Zurich, Switzerland

E. Petelenz-Kurdziel · D. Klein · S. Hohmann

Department of Cell and Molecular Biology/Microbiology,

University of Gothenburg, Box 462, 40530 Göteborg, Sweden about these concepts using a model discrimination approach. In accordance with previous reports, we estimated an average initial turgor of $0.6 \pm 0.2 \mathrm{MPa}$ and found that turgor becomes negligible at a relative volume of $93.3 \pm 6.3 \%$ corresponding to an osmotic shock of $0.4 \pm 0.2 \mathrm{Osm} / \mathrm{l}$. At high stress levels $(4 \mathrm{Osm} / \mathrm{l})$, plasmolysis may occur. We found that the volumetric elastic modulus, a measure of cell wall elasticity, is $14.3 \pm 10.4 \mathrm{MPa}$. Our model discrimination analysis suggests that other thermodynamic quantities affecting the intracellular water potential, for example the matrix potential, can be neglected under physiological conditions. The parameterized turgor models showed that activation of the osmosensing high osmolarity glycerol (HOG) signaling pathway correlates with turgor loss in a 1:1 relationship. This finding suggests that mechanical properties of the membrane trigger HOG pathway activation, which can be represented and quantitatively modeled by turgor.

Present Address:

D. Klein

Clinical R\&D Laboratory, Oxoid, Microbiology,

Basingstoke, Thermo Fisher Scientific, Perth, UK

J. Schaber ( $\bowtie)$

Institute for Experimental Internal Medicine, Medical Faculty,

Otto von Guericke University, Leipziger Str. 44,

39120 Magdeburg, Germany

e-mail: schaber@med.ovgu.de

Present Address:

E. Eriksson

SP Technical Research Institute of Sweden,

Brinellgatan 4, Box 857, 50115 Borås, Sweden 
Keywords Turgor - Cell wall elasticity .

Volumetric elastic modulus - High osmolarity glycerol (HOG) signaling · Plasmolysis · Model discrimination · Yeast

\section{Introduction}

All living cells maintain an osmotic pressure gradient between their interior and the extracellular environment. This gradient is counterbalanced by a hydrostatic pressure, called turgor, which is especially prominent in plants and unicellular organisms, most notably fungi. The concept of turgor has been debated for many years (Burström 1971). It is recognized that turgor plays a vital role in growth (Cosgrove 1981; Zimmermann 1978; Marechal and Gervais 1994), cell structure (Cosgrove 1993; Morris et al. 1986; Zhongcan and Helfrich 1987; Munns et al. 1983), and membrane transport processes (Zimmermann 1978; Lande et al. 1995; Kamiya et al. 1963; Soveral et al. 2008) and that it may be sensed by the cells as an indicator of external osmotic changes (Tamas et al. 2000; Coster et al. 1976; Schmalstig and Cosgrove 1988; Reiser et al. 2003). Therefore, quantifying cell wall and membrane properties, and thus the resulting turgor pressure, has been the aim of numerous studies (Cosgrove 1981; Morris et al. 1986; Munns et al. 1983; Kamiya et al. 1963; Coster et al. 1976; Smith et al. 1998, 2000a, b, c; Marechal et al. 1995; Gervais et al. 1996a; Cosgrove 2000). Parameterized models of turgor pressure, based on cell wall and membrane properties are especially important for quantitative mathematical models describing the interdependence between water transport and signaling processes (Schaber and Klipp 2008; Klipp et al. 2005; Gennemark et al. 2006; Kargol and Kargol 2003a, 2003).

At present, it is not possible to directly measure turgor in yeast. However, the so-called linear elastic theory states that the change in turgor pressure $P$ is proportional to a relative change in membrane enclosed cell volume $V_{\mathrm{m}}$, i.e.,

$\mathrm{d} P=\varepsilon \frac{\mathrm{d} V_{m}}{V_{m}}$,

where $\varepsilon$ is a proportionality factor, called volumetric elastic modulus or bulk modulus. This factor can be viewed as a measure of the elasticity of the cell wall. Thus, the turgor pressure can be derived from cell volume measurements that have been performed repeatedly on yeast cells under different experimental conditions (Munns et al. 1983; Smith et al. 2000a, b, c; Touhami et al. 2003; Wei et al. 2001; Martinez de Maranon et al. 1996; Arnold and Lacy 1977). However, the results obtained for turgor pressure were highly inconsistent, with variations spanning up to three orders of magnitude from 0.05 (Martinez de Maranon et al. 1996) to 2.9 (Arnold and Lacy 1977) MPa.

The concepts of turgor, cell wall elasticity, osmotically active volume and intracellular osmolarity have never been quantified and parameterized together in a consistent framework, nor challenged by alternative concepts (Smith et al. 2000c; Martinez de Maranon et al. 1996; Gervais et al. 1996b). Moreover, the few reports in which turgor and/or cell wall elasticity were quantitatively determined used non-physiological experimental conditions. For example, one study used pre-stressed cells (Marechal et al. 1995) and in two others, the cells were suspended in distilled water before volume measurements (Smith et al. 2000b, c).

The objective of this study was to derive parameterized models of turgor and other biophysical properties of the cell, for example the volumetric elastic modulus, in Saccharomyces cerevisiae. We developed a modeling framework that includes the concepts of turgor pressure, intra and extracellular osmotic pressure, and membrane and volume properties and, for the first time, also other thermodynamic quantities, for example the matrix potential, in a consistent manner. By reformulating sub-modules while keeping the overall framework consistent, we could test several hypotheses describing the relationship between cell volume and changes in the intracellular water potential. Verification of these hypotheses was based on four independent data sets. To ensure a wide applicability of the selected model, the conditions for generating each data set were slightly different. We also correlated cell volume measurements with Hog1 phosphorylation levels and Hog1-GFP nuclear localization to investigate the physiological consequences of turgor loss under hyper-osmotic stress in a quantitative fashion. Hog 1 is the yeast p38 stress-activated protein kinase whose phosphorylation level and subcellular localization are controlled by an osmo-sensing signaling pathway (Hohmann 2002). We found a 1:1 relationship between turgor loss and HOG pathway activation. A loss of turgor relates to HOG pathway activation such that when turgor becomes zero because of application of a hyperosmotic solution the HOG pathway is maximally activated. This finding suggests that the extent of HOG pathway activation is directly linked to some mechanical property of the membrane, which can be represented and quantitatively modeled by the loss of turgor pressure.

\section{Materials and methods}

Descriptions and units of all variables and parameters are listed in Table 1. 
Table 1 Summary of quantities used in the main text

\begin{tabular}{|c|c|c|}
\hline Quantity & Unit & Description \\
\hline$V_{\text {ap }}$ & $\%$ initial volume & Apparent cell volume, enclosed by the cell wall \\
\hline$V_{\mathrm{ap}}^{0}$ & $\%$ initial volume & Initial $V_{\text {ap }}$ \\
\hline$V_{\mathrm{ap}}^{\min }$ & $\%$ initial volume & Minimal $V_{\text {ap }}$ attained after hyperosmotic shock \\
\hline$V_{\mathrm{m}}$ & $\%$ initial volume & Cell volume, enclosed by the plasma membrane \\
\hline$V_{\mathrm{os}}$ & $\%$ initial volume & Osmotically active volume \\
\hline$V_{\mathrm{pl}}$ & $\%$ initial volume & $\begin{array}{l}\text { Periplasmic volume. We tested three alternative models of } V_{\mathrm{pl}} \text { as a function of } c_{\text {stress }}^{\mathrm{e}} \text { (see } \\
\text { "Supplementary material") }\end{array}$ \\
\hline$V_{\mathrm{b}}$ & $\%$ initial volume & Solid cytosolic volume \\
\hline$V_{\mathrm{m}}^{P=0}$ & $\%$ initial volume & Volume, when turgor becomes zero \\
\hline$V_{\mathrm{m}}^{\tau}$ & $\%$ initial volume & Volume below which negative hydrostatic or matrix potential effects are important \\
\hline$V_{\mathrm{ap}}^{\mathrm{pl}}$ & $\%$ initial volume & Apparent volume where plasmolysis occurs \\
\hline$c_{0}^{i}$ & Osm/l & Initial internal osmolarity. \\
\hline$c_{0}^{\mathrm{e}}$ & Osm/l & Initial external osmolarity \\
\hline$c_{\text {stress }}^{\mathrm{e}}$ & Osm/l & Applied osmotic stress \\
\hline$c_{P=0}^{\mathrm{e}}$ & Osm/l & $c_{\text {stress }}^{\mathrm{e}}$ corresponding to $V_{\mathrm{m}}^{P=0}$ \\
\hline$c_{\tau}^{\mathrm{e}}$ & Osm/l & $c_{\text {stress }}^{\mathrm{e}}$ corresponding to $V_{\mathrm{m}}^{\tau}$ \\
\hline$c_{\mathrm{pl}}^{\mathrm{e}}$ & Osm/l & $c_{\text {stress }}^{\mathrm{e}}$ corresponding to $V_{\mathrm{ap}}^{\mathrm{pl}}$ \\
\hline$P_{0}$ & $\mathrm{MPa}$ & Initial turgor at the initial turgid cell volume of $100 \%$ \\
\hline$P_{V_{\mathrm{ap}}^{\min }}$ & $\mathrm{MPa}$ & $\begin{array}{l}\text { Turgor at } V_{\mathrm{ap}}^{\min } \text {. In general, there are two alternatives, i.e., the one-sided model (Eq. S7 in } \\
\text { "Supplementary material") and the two-sided model (Eq. S8 in "Supplementary material") } \\
\text { (Fig. 2) }\end{array}$ \\
\hline$\varepsilon$ & $\mathrm{MPa}$ & $\begin{array}{l}\text { Volumetric elastic modulus, describing the elasticity of the cell wall, relating relative volume } \\
\text { change to hydrostatic pressure, i.e., turgor }\end{array}$ \\
\hline$\varepsilon_{\tau}$ & $\mathrm{MPa}$ & $\begin{array}{l}\text { Proportionality factor, relating relative volume change to the matrix potential or other effects } \\
\text { negatively influencing the intracellular water potential }\end{array}$ \\
\hline$R$ & $\mathrm{~J} \mathrm{~K}^{-1} \mathrm{~mol}^{-1}$ & Gas constant \\
\hline$T$ & $\mathrm{~K}$ & Temperature \\
\hline$\alpha_{\mathrm{PC}}$ & Dimensionless & Conversion factor relating pressure to osmolarity, e.g., for relating $\mathrm{MPa}$ and Osm/l it is $10^{-3}$ \\
\hline
\end{tabular}

\section{Modeling framework}

We assume that the change in osmotically active volume $V_{\text {os }}$ is equal to the water flux across the cell membrane, which is driven by differences in chemical water potentials inside and outside the cell. Based on these thermodynamic principles (for details refer to the "Supplementary material") we developed a model describing the minimal volume of yeast cells attained after osmotic shock, $V_{\mathrm{ap}}^{\mathrm{min}}$, which included several new aspects that are depicted in Fig. 1.

We made a distinction between the commonly measured volume that is enclosed by the cell wall, which we refer to as the apparent volume $V_{\text {ap }}$, and the membrane-enclosed cytoplasmic volume, $V_{\mathrm{m}}$, where osmotic and hydrostatic pressures are defined. $V_{\mathrm{m}}$ comprises the osmotically active volume $V_{\text {os }}$ and the minimal solid cytosolic volume $V_{\mathrm{b}}$, i.e., $V_{\mathrm{m}}=V_{\mathrm{os}}+V_{\mathrm{b}}$. We assume that for osmotic shocks higher than a critical value of $c_{\mathrm{pl}}^{\mathrm{e}}$ or volumes lower than a critical value of $V_{\mathrm{ap}}^{\mathrm{pl}}$, the membrane $\left(V_{\mathrm{m}}\right)$ detaches from the cell wall $\left(V_{\mathrm{ap}}\right)$, i.e., plasmolysis occurs, giving rise to the periplasmic volume $V_{\mathrm{pl}}$, such that $V_{\mathrm{ap}}=V_{\mathrm{m}}+V_{\mathrm{pl}}$. Furthermore, we define $V_{\mathrm{m}}^{P=0}$ as the volume at which the turgor becomes zero and $V_{\mathrm{m}}^{\tau}$ as the volume where negative hydrostatic effects and/or matrix potential effects become important, corresponding to hyperosmotic shock levels of $c_{P=0}^{\mathrm{e}}$ and $c_{\tau}^{\mathrm{e}}$, respectively (Fig. 1). The latter two are thresholds that affect the rate of water outflow, i.e., shrinkage. Between $V_{\mathrm{m}}^{P=0}$ and $V_{\mathrm{m}}^{\tau}$ apparent cell volume is a reciprocal function of external osmolarity, also called the van't Hoff relationship (Noble 1969) (Eq. S6 in "Supplementary material"). Based on these concepts, we derived a formula for the apparent minimum volume after osmotic shock $V_{\mathrm{ap}}^{\mathrm{min}}$ as a function of turgor pressure $P_{V_{\mathrm{ap}}^{\min }}$, osmolarities and volumes:

$$
V_{\mathrm{ap}}^{\min }=V_{\mathrm{b}}+V_{\mathrm{pl}}+\frac{c_{0}^{i}\left(V_{\mathrm{ap}}^{0}-V_{\mathrm{b}}-V_{\mathrm{pl}}\right)}{\frac{P_{V_{\mathrm{ap}}^{\min }}}{\alpha_{\mathrm{pC}} R T}+c_{\text {stress }}^{\mathrm{e}}+c_{0}^{\mathrm{e}}}
$$

where $c_{0}^{i}$ is the initial internal osmolarity (Eq. S4 in "Supplementary material"), $V_{\mathrm{ap}}^{0}$ is the initial apparent cell 

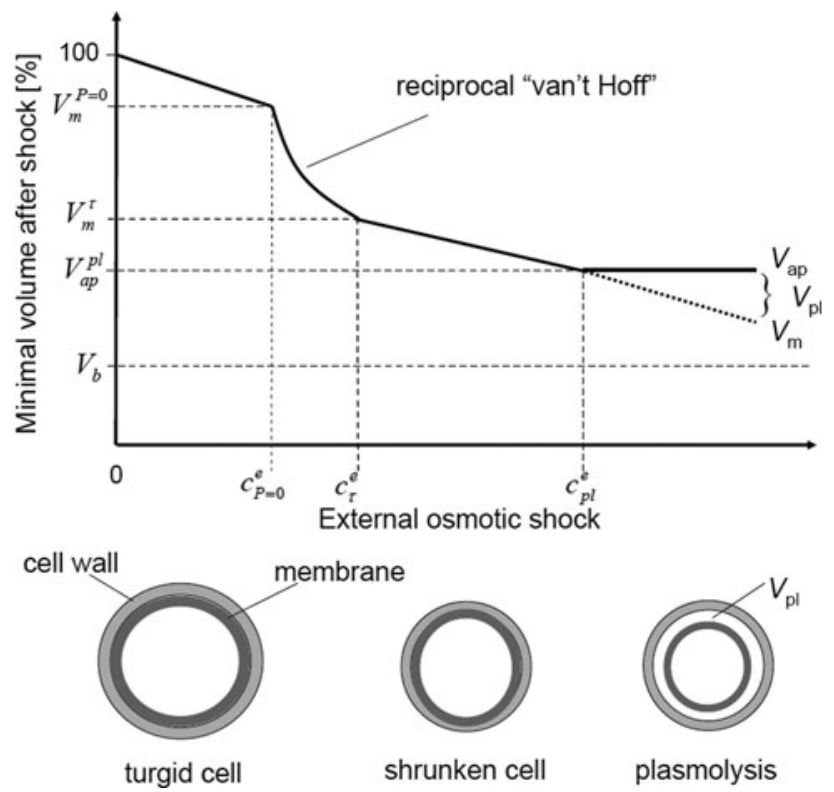

Fig. 1 Qualitative representation of cell volume concepts. Solid line: $V_{\text {ap }}$, the apparent cell wall enclosed volume. Dotted line: $V_{\mathrm{m}}$, the membrane enclosed volume. $V_{\mathrm{m}}^{P=0}$ is the volume at which the turgor becomes zero and $V_{\mathrm{m}}^{\tau}$ is the volume where negative hydrostatic effects or matrix potential effects become important, corresponding to hyperosmotic shock levels of $c_{P=0}^{\mathrm{e}}$ and $c_{\tau}^{\mathrm{e}}$, respectively. $c_{\mathrm{pl}}^{\mathrm{e}}$ and $V_{\mathrm{ap}}^{\mathrm{pl}}$ are the stress and volume thresholds, respectively, where $V_{\mathrm{m}}$ detaches from $V_{\mathrm{ap}} . V_{\mathrm{pl}}$ is the periplasmic volume. $V_{\mathrm{b}}$ is the minimum solid cytosolic volume. Note, that it is not necessarily true that $c_{\tau}^{\mathrm{e}}<c_{\mathrm{pl}}^{\mathrm{e}}$ and $V_{\mathrm{m}}^{\tau}>V_{\mathrm{ap}}^{\mathrm{pl}}$. The diagrams of the cells illustrate the cell wall and the membrane in different states after hyper-osmotic shock

volume, $R$ is the gas constant, $T$ is the temperature, $\alpha_{\mathrm{PC}}$ is a dimensionless conversion factor, $c_{0}^{\mathrm{e}}$ is the initial external osmolarity, and $c_{\text {stress }}^{\mathrm{e}}$ is the applied osmotic stress.

Turgor pressure and matrix potential

Thermodynamic quantities other than turgor and osmolarity, that can affect the cellular water potential, are usually ignored. For example, a specific fraction of the intracellular water might be bound to colloidal surfaces by electrostatic interactions. This water is not freely available in solution, which lowers the total intracellular water potential by a value that is termed the matrix potential (Griffin 1981). In addition, it is possible that the cytoskeleton mechanically counteracts cell shrinkage such that the cell has an inherent tendency to re-swell in a sponge-like fashion. This effect would also reduce the total intracellular water potential. For simplicity, we summarize these effects under the term matrix potential $(\Delta \tau$ in Eq. S1 of the "Supplementary material"). Quantitative descriptions of the matrix potential are not available and elaborated models of mechanical forces induced by volume changes (Stamenovic and

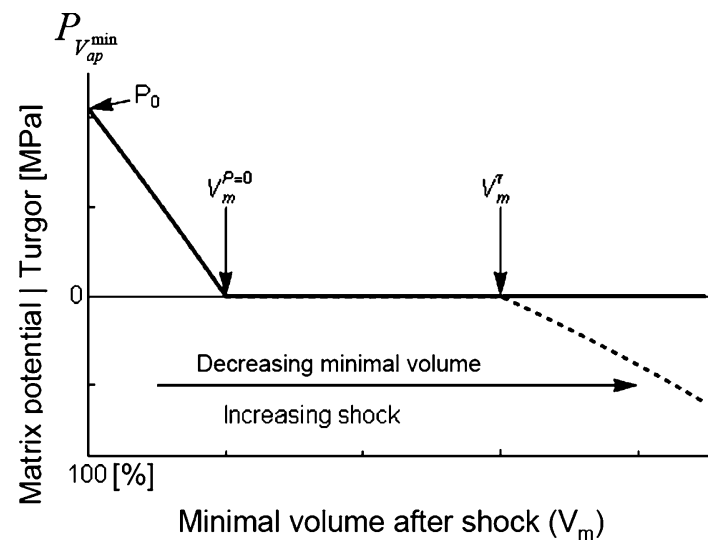

Fig. 2 Illustration of turgor and matrix potential. Turgor (black line), which has an initial value of $P_{0}$ at the initial turgid cell volume of $100 \%$, is displayed on the positive $y$-axis. When the cell shrinks, the turgor decreases to zero at the volume $V_{\mathrm{m}}^{P=0}$. This is referred to as the "one-sided turgor model". The matrix potential or other effects that reduce the water potential are displayed on the negative $y$-axis. These are assumed to become important below a specific threshold volume $V_{\mathrm{m}}^{\tau}$. The model represented by the solid line up to $V_{\mathrm{m}}^{\tau}$ together with the dashed line is referred to as the "two-sided turgor model"

Coughlin 1999) are beyond the scope of this study. Therefore, we include the effects summarized under the term of the matrix potential in the model of the turgor pressure $P_{V_{\mathrm{ap}}^{\min }}$ with a simple mathematical description. In this way, we obtain an expression for $P_{V_{\text {ap }}^{\min }}$ including the matrix potential (assuming $V_{\mathrm{pl}}=0$ for $V_{\mathrm{m}} \geq V_{\mathrm{m}}^{P=0}$ ):

$$
\begin{aligned}
& P_{V_{\mathrm{ap}}^{\min }}\left(V_{\mathrm{ap}}^{\min }, V_{\mathrm{pl}}, V_{\mathrm{m}}^{P=0}, V_{\mathrm{m}}^{\tau}, \varepsilon, \varepsilon_{\tau}\right) \\
& =\left\{\begin{array}{lll}
\varepsilon \ln \left(\frac{V_{\mathrm{p}}^{\min }}{V_{\mathrm{m}}^{P=0}}\right) & \text { for } \quad V_{\mathrm{ap}}^{\min } \geq V_{\mathrm{m}}^{P=0} \\
0 & \text { for } & V_{\mathrm{m}}^{\tau} \leq V_{\mathrm{ap}}^{\min }-V_{\mathrm{pl}}<V_{\mathrm{m}}^{P=0} \\
\varepsilon_{\tau} \ln \left(\frac{V_{\mathrm{ap}}^{\min }-V_{\mathrm{pl}}}{V_{\mathrm{m}}^{\tau}}\right) & \text { for } & V_{\mathrm{ap}}^{\min }-V_{\mathrm{pl}}<V_{\mathrm{m}}^{\tau}
\end{array}\right.
\end{aligned}
$$

In Fig. $2 P_{V_{\text {ap }}^{\min }}$ is displayed as a function of $V_{\mathrm{m}}=V_{\mathrm{ap}}^{\mathrm{min}}-V_{\mathrm{pl}}$

We tested Eq. 3 against the data, with and without the term for matrix potential, which we refer to as the onesided and the two-sided models, respectively. We also tested three hypotheses regarding the size of the periplasmic volume $V_{\mathrm{pl}}$, (a) $V_{\mathrm{pl}}=0$, (b) $V_{\mathrm{pl}}>0$ with a constant apparent volume $V_{\mathrm{ap}}^{\mathrm{pl}}$ (Fig. 1; Eq. S10 in "Supplementary material"), and (c) $V_{\mathrm{pl}}>0$ with a variable apparent volume $V_{\mathrm{ap}}^{\mathrm{pl}}$ (Eq. S11 in "Supplementary material").

Inserting Eq. 3 into Eq. 2 yields an implicit expression for $V_{\mathrm{ap}}^{\mathrm{min}}$ that depends on various parameters of the specific model for turgor, matrix effect, and periplasmic volume. When a solution exists, this solution is unique (see "Supplementary material"). 
Model discrimination

Using Eq. 2 together with a model for $V_{\mathrm{pl}}$ (Eqs. S10, S11 in "Supplementary material") and a turgor model (Eq. 3; Eqs. S7, S8 in "Supplementary material"), a solution for $V_{\mathrm{ap}}^{\min }$ can be numerically determined. When $V_{\mathrm{ap}}^{\min }$ is measured, Eq. 2 can be fitted to the data by estimating the respective parameters (Table S1 in "Supplementary material"), e.g., by minimizing the sum of squared residuals (SSR)

$\operatorname{SSR}(x)=\sum_{i=1}^{n} \frac{1}{\sigma_{i}}\left(V_{\mathrm{ap}, i}^{\mathrm{min}}(x)-V_{\text {measured }, i}^{\mathrm{min}}\right)^{2}$,

where $x=\left(x_{1}, x_{2}, \ldots, x_{\mathrm{m}}\right)$ denote the parameters that are to be estimated and that depend on the model (Table S1 in "Supplementary material"), $\sigma_{\mathrm{i}}$ is the standard deviation of the measurements and $n$ is the number of data points. For the sake of simplicity, we assume that the measurement errors are independently normally distributed. Thus, the SSR becomes a maximum-likelihood estimator and we can use the Akaike's information criterion corrected for small sample size (AICc) to rank and, thus, discriminate the models according to their SSR and number of parameters (Burnham and Anderson 2002):

$\mathrm{AICc}=2 k+n\left(\ln \left(\frac{2 \pi \mathrm{SSR}}{n}\right)+1\right)+\frac{2 k(k+1)}{n-k-1}$,

where $k$ is the number of parameters.

The fitting was performed by differential evolution, a global optimization method (Storn and Price 1997), and the models were ranked according to the AICc (Eq. 4). Additionally, for the best model, we performed a MonteCarlo analysis by re-sampling the data a hundred times assuming a normal distribution with the respective standard deviations for each data point. In this way, means and confidence intervals for the estimated parameters could be obtained.

\section{Data sets}

We collected three independent data sets (data sets $1-3$ in Fig. 3; Fig. S1 in "Supplementary material") and took one data set from the literature (Marechal et al. 1995) (data set 4 in Fig. 3; Fig. S2 in "Supplementary material"). The cell volume was quantified immediately before and after the hyperosmotic stress, ensuring that the minimum volume after osmotic shock was measured. For our experiments we used $\mathrm{NaCl}$ as an osmoticum, whereas in the data set from literature yeast cells were subjected to a wide range of glycerol concentrations (refer to Marechal et al. 1995 for details). Table 2 summarizes the different data sets. Experimental details for data sets 1-3 are described in the "Supplementary material".

\section{Results}

Parameter fitting and model selection predicts turgor pressure and other biophysical cell properties

We fitted $V_{\mathrm{ap}}^{\min }$ from Eq. 2 to four different data sets using alternative models for $P_{V_{\mathrm{m}}^{\min }}$ and $V_{\mathrm{p}}$, resulting in seven different models (see Table S1 in "Supplementary material" for a complete listing). For each data set the best model according to the AICc is described in Table 3. The fitted and measured $V_{\mathrm{ap}}^{\min }$ are displayed in Fig. 3, with the corresponding predicted turgor pressure.

One-sided turgor model is most appropriate for physiological stress conditions

The three data sets produced for this study were all fitted best by the one-sided turgor model based on Eqs. 1 and 3, where only the positive turgor pressure plays a role (Fig. 2). Even though this is the generally accepted model (Cosgrove 1981; Marechal and Gervais 1994; Klipp et al. 2005; Gennemark et al. 2006; Meikle et al. 1988), we tested it with six other models, because to our knowledge it has never been challenged by other models, nor rigorously fitted to data. Thus, we show for the first time that effects resisting cell shrinkage, for example the matrix potential or mechanical forces, can be neglected under physiological conditions. Consequently, when the turgor becomes zero after an external osmotic shock of $c_{P=0}^{\mathrm{e}}$ (Table 1; Fig. 3), the measured apparent volume $V_{\text {ap }}$ is well predicted by the membrane enclosed volume $V_{\mathrm{m}}$ according to the van't Hoff's equation (Eq. S6 in "Supplementary material"). This means that the cell wall tightly follows the shrinkage of the membrane enclosed volume. Data set 4 , which was collected by applying extreme stress levels, also favored the one-sided turgor model. However, at $4 \mathrm{Osm} / \mathrm{l}$ plasmolysis was predicted, with a constant minimal apparent volume $V_{\mathrm{ap}}^{\mathrm{pl}}$ of $65 \%$.

Estimated biophysical cell properties are similar between experiments

Parameters estimated on the basis of the datasets give a consistent picture of important biophysical cell properties. For data sets 2 and 3, where single cell information was available, we distinguished between small and large cells and repeated the analysis to test whether cell size had an effect on the results. We found no significant differences between small and large cells with regard to the estimated parameters, which is in agreement with earlier reports (Martinez de Maranon et al. 1996). The solid volume $V_{\mathrm{b}}$ was estimated to be between 33 and $49 \%$ of the initial cell volume, which also corresponds to earlier reports 

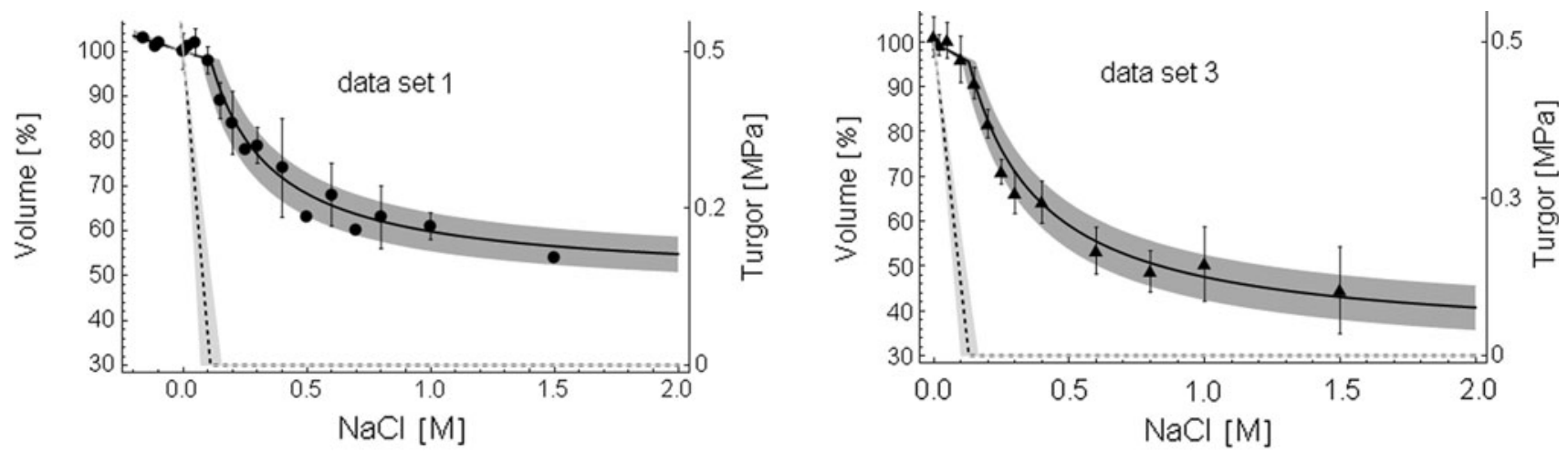

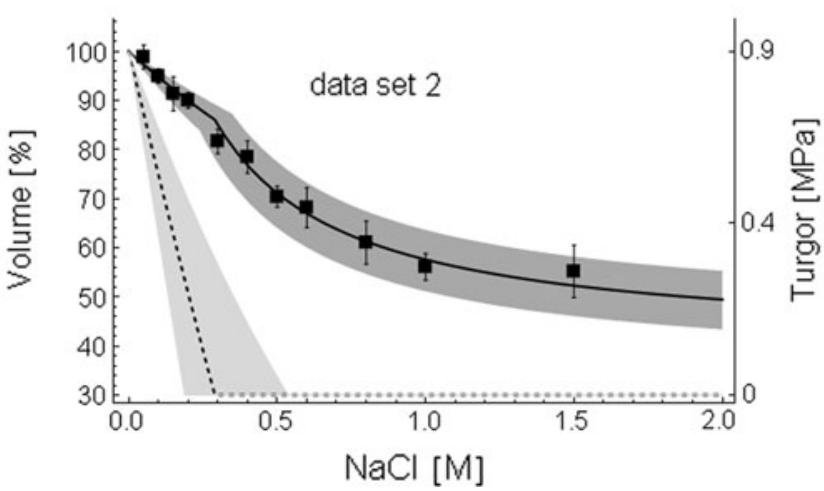

Fig. 3 Best fit models and data. The data and the best fit model (solid line) refer to the left $y$-axis. The dark gray region depicts the $95 \%$ confidence region of the fitted model. The right $y$-axis refers to the estimated turgor pressure (dashed line). The light gray region depicts the $95 \%$ confidence region of the estimated turgor pressure.

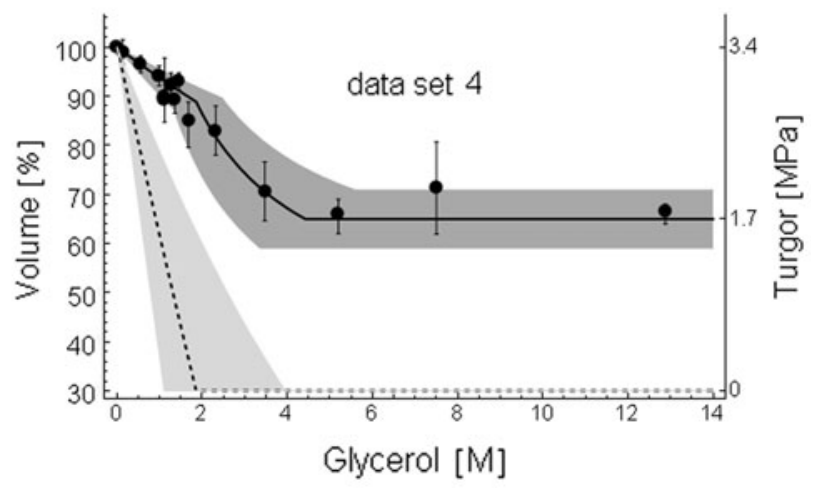

The $x$-axis refers to concentrations of the respective stress agent. The confidence regions were calculated on the basis of the confidence intervals of the estimated parameters (Table 3). The four data sets are described in the "Methods" sections and the "Supplementary material"

Table 2 Data sets used for model discrimination

\begin{tabular}{llllllllll}
\hline No. & Method & Strain & Medium & Agent & ${ }^{\circ} \mathrm{C}$ & Growth & $c_{0}^{\mathrm{e}}(\mathrm{Osm} / \mathrm{l})$ & $\# n$ & Refs. \\
\hline 1 & Particle sizer & $\mathrm{W} 303$ & YPD & $\mathrm{NaCl}$ & 30 & Early log & 0.26 & 16 & This study \\
2 & Single cells bright field & $\mathrm{BY} 4741$ & YNB & $\mathrm{NaCl}$ & 20 & Log & 0.27 & 11 & This study \\
3 & Single cells fluorescence & W303 & SD & $\mathrm{NaCl}$ & 30 & Log & 0.25 & 13 & This study \\
4 & Single cells bright field & CBS1171 & Wickerham & Glycerol & 25 & Stationary & 0.86 & 15 & Marechal et al. (1995)
\end{tabular}

$c_{0}^{\mathrm{e}}$ was measured for data sets 1-3 with an osmometer and calculated for data set 4 from Marechal et al. (1995), \# is the number of data points

(Marechal et al. 1995; Meikle et al. 1988). The initial turgor estimated on the basis of the three data sets with similar experimental conditions (data sets 1-3) was $0.6 \pm 0.2 \mathrm{MPa}$ on average, which confirmed results from earlier studies (Smith et al. 2000c; Gervais et al. 1996a; Meikle et al. 1988). The estimated membrane rigidity $\varepsilon$ was the parameter that varied the most between experiments and it turned out to be a sensitive, poorly determined parameter, which is reflected by the relatively high standard deviations. However, at least for the three directly comparable data sets $1-3$, the results were similar, giving an average volumetric elastic modulus of $14.3 \pm 10.4 \mathrm{MPa}$ ranging from 5.7 to $25.8 \mathrm{MPa}$.
On the basis of our general modeling framework and the estimated parameters, we could derive several other interesting cell properties. The initial osmolarity within the cell $c_{0}^{i}$ was estimated to be up to more than twice the osmolarity of the outside medium. Thus, apparently, yeast can maintain substantial osmotic gradients between the inside and outside of the cell, which are equilibrated by turgor. The volume at which turgor becomes zero was estimated to be of $93.3 \pm 6.3 \%$ on average for data sets $1-3$. The corresponding external shock for the salt experiments was $0.4 \pm 0.2 \mathrm{Osm} / \mathrm{l}$, corresponding to a salt concentration of roughly $0.2 \mathrm{M} \mathrm{NaCl}$. As this is within the range of concentrations in which saturation of HOG pathway activation 
Table 3 Results of parameter fits of Eq. 2 for the best selected model according to the AICc

\begin{tabular}{|c|c|c|c|c|c|}
\hline & Data set 1 & Data set 2 & Data set 3 & Data set 4 & \\
\hline$\#$ & 2 & 2 & 2 & 4 & Selected model \\
\hline$P_{V_{\mathrm{m}}^{\min }}$ & One-sided & One-sided & One-sided & One-sided & \\
\hline$V_{\mathrm{pl}}$ & 0 & 0 & 0 & Constant $V_{\mathrm{ap}}^{\mathrm{pl}}$ & \\
\hline$k$ & 3 & 3 & 3 & 4 & \\
\hline$V_{\mathrm{b}}$ & $49.0 \pm 3.4$ & $40.2 \pm 5.5$ & $33.0 \pm 4.8$ & $40.1 \pm 11.1$ & Estimated parameters \\
\hline$P_{0}$ & $0.5 \pm 0.1$ & $0.9 \pm 0.2$ & $0.5 \pm 0.1$ & $3.4 \pm 1.0$ & \\
\hline$\varepsilon$ & $25.8 \pm 7.0$ & $5.7 \pm 2.0$ & $11.4 \pm 2.1$ & $28.6 \pm 11.3$ & \\
\hline$V_{\mathrm{ap}}^{\mathrm{pl}}$ & & & & $65.0 \pm 5.9$ & \\
\hline$c_{0}^{i}$ & $0.4 \pm 0.1$ & $0.6 \pm 0.1$ & $0.5 \pm 0.0$ & $2.2 \pm 0.4$ & Derived parameters \\
\hline$V_{\mathrm{m}}^{P=0}$ & $98.2 \pm 3.1$ & $86.1 \pm 6.3$ & $95.5 \pm 6.8$ & $88.9 \pm 10.8$ & \\
\hline$c_{P=0}^{\mathrm{e}}$ & $0.2 \pm 0.1$ & $0.5 \pm 0.2$ & $0.2 \pm 0.1$ & $1.9 \pm 1.9$ & \\
\hline$c_{\mathrm{pl}}^{\mathrm{e}}$ & & & & $4.4 \pm 2.2$ & \\
\hline
\end{tabular}

For a complete description of parameters and units refer to Table 1

\# Number of best selected model (Table $\mathrm{S} 1$ in "Supplementary material"), $P_{V_{\mathrm{m}} \min }$ : selected turgor model (Fig. 2; Eq. 3), $V_{\mathrm{pl}}$ : selected $V_{\mathrm{pl}}$ model (Fig. 1; Eq. S10 in "Supplementary material"), $k$ : number of fitted parameters, $V_{\mathrm{b}}$ : solid base volume, $P_{0}$ : initial turgor, $\varepsilon$ : volumetric elastic modulus, $V_{\mathrm{ap}}^{\mathrm{pl}}$ apparent volume where plasmolysis occurs, $c_{0}^{i}$ : initial internal osmolarity, $V_{\mathrm{m}}^{P=0}$ : volume at zero turgor, $c_{P=0}^{e}$ : applied external stress to equilibrate turgor, $c_{\mathrm{pl}}^{e}$ : applied external stress to reach $V_{\mathrm{ap}}^{\mathrm{pl}}$. Estimated parameters are given as mean \pm standard deviation from 100 fits where the data points were sampled from their respective standard deviations indicated in Figs. 3 and 4 . The mean of parameters in the lower part are derived from the mean parameters in the upper part

is observed (Maeda et al. 1995), we hypothesized that the initial HOG pathway response may be correlated with turgor.

Loss of turgor correlates with HOG pathway activation

The exact biophysical mechanisms that trigger the activation of the HOG pathway in yeast have not yet been determined (Hohmann 2002), but there is evidence that the turgor is one of the factors involved (Tamas et al. 2000; Reiser et al. 2003). As our computational analysis provided us with parameterized models of turgor pressure (Fig. 3; Table 3), we tested whether there is a correlation between the loss of turgor for different degrees of osmotic shock and the extent of HOG pathway activation. Thus, in addition to cell volume measurements, we collected two independent data sets reflecting the degree of HOG pathway activation depending on the intensity of the hyperosmotic stress. In the first data set, the extent of Hog1 nuclear accumulation was measured, whereas the second data set represents the extent of Hog1 phosphorylation in response to different $\mathrm{NaCl}$ concentrations (Fig. 4, for details about the measurements refer to the "Supplementary material").

By considering both Hog1 activation (Fig. 4) and the predicted turgor (dotted line in Fig. 3) as a function of applied osmotic stress, we could investigate Hog1 activation as a function of predicted turgor (Fig. 5). With three different parameterizations for the turgor model (from data sets 1-3, data set 4 was disregarded, because it used a different stress agent) and two different $\operatorname{Hog} 1$ activation

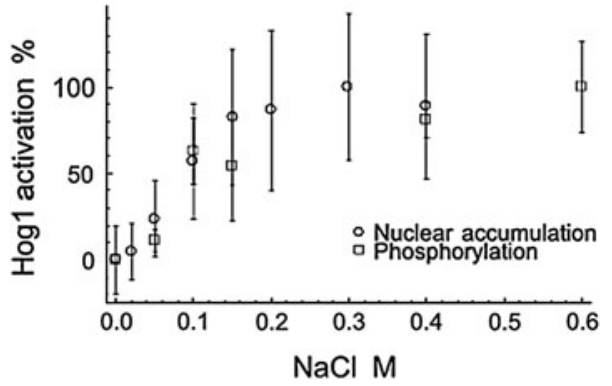

Fig. 4 Hog 1 activation data. Circles: maximum Hog1 nuclear concentration after osmotic shock. Squares: Hog1 phosphorylation after $2 \mathrm{~min}$ of shock treatment. Data are scaled to the respective measured maximum

data sets, we obtained six turgor-Hog1 activation correlations, of which we display three in Fig. 5. The others are displayed in the "Supplementary material" (Fig. S3).

We fitted a linear relationship between HOG pathway activation and relative turgor for the three different parameterized models, corresponding to data sets $1-3$, and the two different HOG pathway activation data sets (black lines in Fig. 5) by a weighted orthogonal regression. Our null hypothesis claimed a direct 1:1 linear relationship, i.e., $\mathrm{H}_{0}: a+b x$, with $(a, b)=(100,-1)$ (gray lines in Fig. 5). To test $\mathrm{H}_{0}$ we computed confidence regions for the estimated parameter pairs $(a, b)$ by a Monte-Carlo analysis. As can be seen from the insets in Fig. 5, in all cases the hypothesized relationship (gray points) was within the $95 \%$ confidence region of the actual estimated parameter pair. Thus, the null hypothesis could not be 

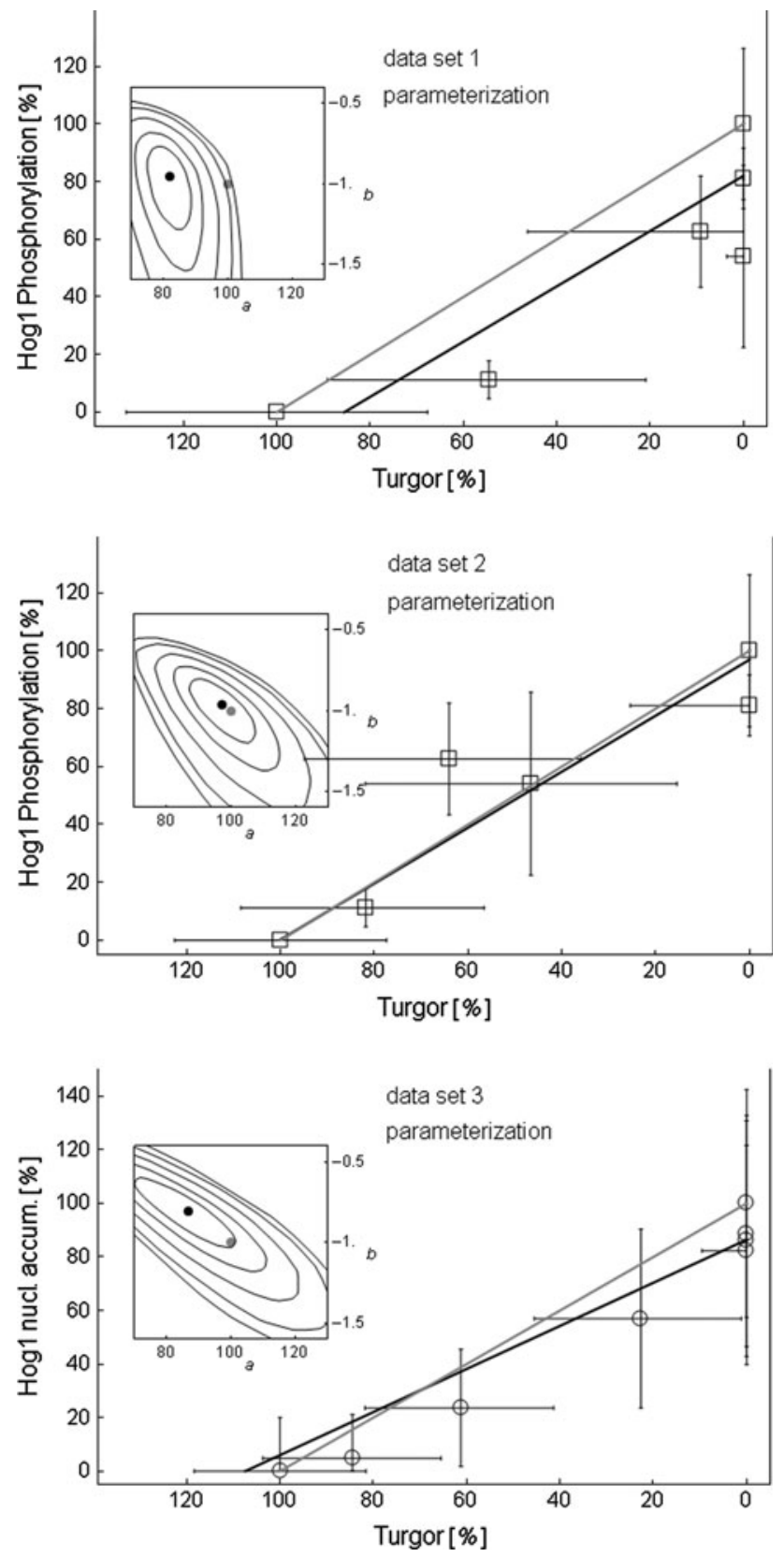

Fig. 5 Turgor-HOG pathway activation relationship. The $x$-axes are relative turgor $(\%)$ as predicted by the parameterized models for different shocks of $\mathrm{NaCl}$ (dotted line in Fig. 3). The $y$-axes are relative HOG pathway activation (\%) according to different shocks of $\mathrm{NaCl}$ (Fig. 4). The black lines are a fitted linear relationship $(y=a+b x)$ based on a weighted orthogonal regression. The gray lines represent the null hypothesis $\mathrm{H}_{0}: y=100-x$, i.e., a direct 1:1 linear relationship between relative loss of turgor and relative HOG pathway activation. The insets are plots of the $(25,50,75$, $90,95 \%)$-confidence regions of the respective estimated parameter pair $(a, b)$ of $a+b x$ with the outermost line being the $95 \%$ confidence region. The black points correspond to the black lines in the plots, the gray points correspond to the gray lines. The confidence regions are obtained by a Monte-Carlo analysis with 1,000 runs rejected. Moreover, the Kendall-rank correlation coefficient (Kendall and Gibbons 1990) was highly significant in all cases $(P<0.01)$.

\section{Discussion}

Parameterized models of biophysical and mechanical cell properties become increasingly important for quantitative and predictive descriptions of cellular behavior. Our modeling framework, which included the concepts of turgor, membrane elasticity, intracellular osmolarity, osmotically active volume, non-turgid volume, and solid cytosolic volume in a coherent and thermodynamically accurate way, enabled us to parameterize all these concepts consistently, by fitting the relevant parameters to experimental data. In addition, it enabled us to discriminate between alternative hypotheses for turgor and volume changes by including alternative sub-models within the general framework. Even though the data sets were based on very different techniques, the overall conclusions are strikingly similar indicating that the proposed mathematical description is both accurate and universal.

We confirmed previous reports, which suggested an initial turgor pressure of around 0.6 $\mathrm{MPa}$ (Smith et al. 2000b; Meikle et al. 1988). Turgor pressures reported for yeast differ substantially. Part of this variation might be because they have never been rigorously fitted to data. In our study, we obtained similar results for data sets 1-3. However, the initial turgor estimated from data set 4 was sixfold higher. We assume that this discrepancy is caused by different growth phases of yeast cells employed in the experiments. This explanation is supported by earlier reports, in which differences in turgor between cells in different physiological states were observed. Specifically, a higher turgor pressure was reported for cells in stationary phase (Smith et al. 2000c; Martinez de Maranon et al. 1996). After examining different possible explanations (Eq. 3), we confirmed the hypothesis that turgor pressure is significant only above a specific volume threshold, below which it can be neglected, as previously proposed (Cosgrove 1981; Marechal and Gervais 1994; Klipp et al. 2005; Gennemark et al. 2006; Meikle et al. 1988). Other forces potentially affecting the water potential, and therefore water flux within the cell, can be neglected under the studied conditions of osmotic shock up to $1.5 \mathrm{M} \mathrm{NaCl}$.

We found values for the volumetric elastic modulus $\varepsilon$ between 6 and $29 \mathrm{MPa}$. We could not find any published values of $\varepsilon$ for yeast. In a recent study, where yeast cells were subjected to mechanical compression, values of 
Young's modulus $E$ for the cell wall of around $110 \mathrm{MPa}$ were reported (Smith et al. 2000a, c). Unfortunately, we could not relate these values to our volumetric elastic modulus, because those values for Young's modulus $E$ were derived assuming a Poisson's ratio $v$ of 0.5 and, hence, the formula $E=3 \varepsilon(1-2 v)$ was not applicable. We noted that cells in stationary phase (data set 4 ) are estimated to be more rigid, i.e., have a higher volumetric elastic modulus, but to a much lesser extent than in Smith et al. (2000c). The cells used in data set 1 were grown to saturation, i.e., beyond proliferation, before they were resuspended in fresh medium for $1 \mathrm{~h}$ before measurements. It is possible that they were still recovering from saturation phase and therefore had higher cell wall rigidity than cells from data sets 2 and 3, which were measured at $\operatorname{logarithmic}$ growth phase. This reflects that $\varepsilon$ is a function of the physiological state of the cell. Thus, when turgor is being modeled over longer time intervals than in this study, the assumption of $\varepsilon$ being constant might be compromised.

Our modeling results support the notion of a rather elastic cell wall, because the cell wall tightly follows the cell membrane on shrinkage, and plasmolysis only occurs under extremely high stresses. There is experimental evidence from mammalian cells that our model of an elastic membrane/cell wall surrounding a viscous medium is an over-simplification (Wang et al. 2001). However, our model proved sufficient to explain our data. More complicated models were not supported by the data in respect of the AICc. It is known that upon osmotic shock the actin cytoskeleton in yeast de-polymerizes (Brewster and Gustin 1994). This is in line with our results in which effects of the skeleton, which we included into the model of the matrix potential, were refuted by the model discrimination. A recent compression study of mammalian cells also ruled out the cytoskeleton as being responsible for the increase in cell stiffness as the volume decreases (Zhou et al. 2009).

Other authors identified the concentration at which plasmolysis occurs as approximately 1 Osm/l (Arnold and Lacy 1977), but under different experimental conditions. The predicted plasmolysis point of $65 \%$ in data set 4 was higher than the lowest volumes measured in the other data sets (44-54\%, Fig. 3). Therefore, cells might have been at the threshold of plasmolysis in those experiments. Because no data were collected at higher stress levels this effect was not distinguishable by model selection.

For the first time, we provide evidence that there is direct link between loss of turgor upon osmotic shock and HOG pathway activation, not only qualitatively but also quantitatively. Moreover, the hypothesis of a direct 1:1 relationship between turgor loss and HOG pathway activation could not be rejected. The physical basis of such a direct relationship remains elusive, but could for example be a change in Sln1 and Sho1 membrane protein conformation as a function of membrane stretch or other mechanical forces that relate to turgor.

Acknowledgments We thank Patrick Gervais for providing a digital version of data set 4, Rosie Perkins, Javier Macia, and Karlheinz Schaber for useful suggestions on the manuscript, and Martina Fröhlich for measurement support. This work was supported via several projects funded by the European Commission: QUASI (Contract No. 503230 to SH, EK, FP and MP), CELLCOMPUT (Contract No. 043310 to SH, EK and FP), UNICELLSYS (Contract No. 201142 to SH, EK, FP, MP and MG), SYSTEMSBIOLOGY (Contract No. 514169 to SH and EK), and AMPKIN (Contract No. 518181 to SH and MG). In addition work was funded by grants from the Swedish Foundation for Strategic Research SSF (Bio-X to MG), the Swedish Research Council (project grants to $\mathrm{SH}$ and $\mathrm{MG}$ ), the Carl Trygger Foundation (to MG), the Science Faculty, University of Gothenburg (to SH and MG), and the Swiss systemsX.ch (to MP).

Open Access This article is distributed under the terms of the Creative Commons Attribution Noncommercial License which permits any noncommercial use, distribution, and reproduction in any medium, provided the original author(s) and source are credited.

\section{References}

Arnold WN, Lacy JS (1977) Permeability of cell-envelope and osmotic behavior in Saccharomyces cerevisiae. J Bacteriol 131:564-571

Brewster JL, Gustin MC (1994) Positioning of cell-growth and division after osmotic-stress requires a MAP kinas pathway. Yeast 10:425-439

Burnham KP, Anderson DR (2002) Model selection and multi-model inference: a practical information-theoretic approach. Springer, Berlin

Burström HG (1971) Wishful thinking of turgor. Nature 234:488

Cosgrove DJ (1981) Analysis of the dynamics and steady-state response of growth-rate and turgor pressure to changes in cell parameters. Plant Physiol 68:1439-1446

Cosgrove DJ (1993) Wall extensibility - its nature, measurement and relationship to plant-cell growth. New Phytol 124:1-23

Cosgrove DJ (2000) Loosening of plant cell walls by expansins. Nature 407:321-326

Coster HGL, Steudle E, Zimmermann U (1976) Turgor pressure sensing in plant-cell membranes. Plant Physiol 58:636-643

Gennemark P, Nordlander B, Hohmann S, Wedelin D (2006) A simple mathematical model of adaptation to high osmolarity in yeast. In Silico Biol 6:193-214

Gervais P, Molin P, Marechal PA, HerailFoussereau C (1996a) Thermodynamics of yeast cell osmoregulation: passive mechanisms. J Biol Phys 22:73-86

Gervais P, Molin P, Marechal PA, Herail-Foussereau C (1996b) Thermodynamics of yeast cell osmoregulation: passive mechanisms. J Biol Phys 22:73-86

Griffin DM (1981) Water and microbial stress. Adv Microb Ecol 5:91-136

Hohmann S (2002) Osmotic stress signaling and osmoadaptation in yeasts. Microbiol Mol Biol Rev 66:300-372

Kamiya N, Tazawa M, Takata T (1963) The relationship of turgor pressure to cell volume in Nitella with special reference to mechanical properties of the cell wall. Protoplasma 57:501-521

Kargol M, Kargol A (2003a) Mechanistic equations for membrane substance transport and their identity with Kedem-Katchalsky equations. Biophys Chem 103:117-127 
Kargol M, Kargol A (2003b) Mechanistic formalism for membrane transport generated by osmotic and mechanical pressure. Gen Physiol Biophys 22:51-68

Kendall M, Gibbons JD (1990) Rank correlation methods. E. Arnold, London

Klipp E, Nordlander B, Kruger R, Gennemark P, Hohmann S (2005) Integrative model of the response of yeast to osmotic shock. Nat Biotechnol 23:975-982

Lande MB, Donovan JM, Zeidel ML (1995) The relationship between membrane fluidity and permeabilities to water, solutes, ammonia, and protons. J Gen Physiol 106:67-84

Maeda T, Takekawa M, Saito H (1995) Activation of yeast PBS2 MAPKK by MAPKKKs or by binding of an SH3-containing osmosensor. Science 269:554-558

Marechal PA, Gervais P (1994) Yeast viability related to water potential variation-influence of the transient phase. Appl Microbiol Biotechnol 42:617-622

Marechal PA, deMaranon IM, Molin P, Gervais P (1995) Yeast cell responses to water potential variations. Int $\mathrm{J}$ Food Microbiol 28:277-287

Martinez de Maranon I, Marechal PA, Gervais P (1996) Passive response of Saccharomyces cerevisiae to osmotic shifts: cell volume variations depending on the physiological state. Biochem Biophys Res Commun 227:519-523

Meikle AJ, Reed RH, Gadd GM (1988) Osmotic adjustment and the accumulation of organic solutes in whole cell and protoplasts of Saccharomyces cerevisiae. J Gen Microbiol 134:3049-3060

Morris GJ, Winters L, Coulson GE, Clarke KJ (1986) Effect of osmotic stress on the on the ultrastructure and viability of the yeast Saccharomyces cerevisiae. J Gen Microbiol 132:20232034

Munns R, Greenway H, Setter TL, Kuo J (1983) Turgor pressure, volumetric elastic-modulus, osmotic volume and ultrastructure of Chlorella emersonii grown at high and low external $\mathrm{NaCl}$. J Exp Bot 34:144-155

Noble PS (1969) The Boyle-Van't Hoff relation. J Theor Biol 23:375-379

Reiser V, Raitt DC, Saito H (2003) Yeast osmosensor Sln1 and plant cytokinin receptor Cre1 respond to changes in turgor pressure. J Cell Biol 161:1035-1040

Schaber J, Klipp E (2008) Short-term volume and turgor regulation in yeast. Essays Biochem 45:147-160

Schmalstig JG, Cosgrove DJ (1988) Growth-inhibition, turgor maintenance, and changes in yield threshold after cessation of solute import in pea epicotyls. Plant Physiol 88:1240-1245
Smith AE, Moxham KE, Middelberg APJ (1998) On uniquely determining cell-wall material properties with the compression experiment. Chem Eng Sci 53:3913-3922

Smith AE, Moxham KE, Middelberg APJ (2000a) Wall material properties of yeast cells. Part II. Analysis. Chem Eng Sci 55:2043-2053

Smith AE, Zhang Z, Thomas CR (2000b) Wall material properties of yeast cells: Part 1. Cell measurements and compression experiments. Chem Eng Sci 55:2031-2041

Smith AE, Zhang ZB, Thomas CR, Moxham KE, Middelberg APJ (2000c) The mechanical properties of Saccharomyces cerevisiae. Proc Natl Acad Sci USA 97:9871-9874

Soveral G, Madeira A, Loureiro-Dias MC, Moura TF (2008) Membrane tension regulates water transport in yeast. Biochim Biophys Acta 1778:2573-2579

Stamenovic D, Coughlin MF (1999) The role of prestress and architecture of the cytoskeleton and deformability of cytoskeletal filaments in mechanics of adherent cells: a quantitative analysis. J Theor Biol 201:63-74

Storn R, Price K (1997) Differential evolution-a simple and efficient heuristic for global optimization over continuous spaces. J Glob Optim 11:341-359

Tamas MJ, Rep M, Thevelein JM, Hohmann S (2000) Stimulation of the yeast high osmolarity glycerol (HOG) pathway: evidence for a signal generated by a change in turgor rather than by water stress. FEBS Lett 472:159-165

Touhami A, Nysten B, Dufrene YF (2003) Nanoscale mapping of the elasticity of microbial cells by atomic force microscopy. Langmuir 19:4539-4543

Wang N, Naruse K, Stamenovic D, Fredberg JJ, Mijailovich SM, Toric-Norrelykke IM, Polte T, Mannix R, Ingber DE (2001) Mechanical behavior in living cells consistent with the tensegrity model. Proc Natl Acad Sci USA 98:7765-7770

Wei CF, Lintilhac PM, Tanguay JJ (2001) An insight into cell elasticity and load-bearing ability. Measurement and theory. Plant Physiol 126:1129-1138

Zhongcan OY, Helfrich W (1987) Instability and deformation of a spherical vesicle by pressure. Phys Rev Lett 59:2486-2488

Zhou EH, Trepat X, Park CY, Lenormand G, Oliver MN, Mijailovich SM, Hardin C, Weitz DA, Butler JP, Fredberg JJ (2009) Universal behavior of the osmotically compressed cell and its analogy to the colloidal glass transition. Proc Natl Acad Sci USA 106:10632-10637

Zimmermann U (1978) Physics of turgor- and osmoregulation. Ann Rev Plant Physiol 29:121-148 